\title{
Raman microspectroscopy imaging analysis of extracellular vesicles (EVs) biogenesis by filamentous fungus Penicilium chrysogenum
}

Ashok Zachariah Samuel, ${ }^{1}$ Shumpei Horii, ${ }^{2,3}$ Takuji Nakashima, ${ }^{1}$ Naoko Shibata, ${ }^{1}$ Masahiro Ando, ${ }^{1}$ Haruko Takeyama ${ }^{* 1,2,4,5}$

1 Research Organization for Nano and Life Innovations, Waseda University, 513, Wasedatsurumaki-cho, Shinjuku-ku, Tokyo 162-0041, Japan

${ }^{2}$ Computational Bio Big-Data Open Innovation Laboratory, AIST-Waseda University, Japan, 34-1 Okubo, Shinjuku-ku, Tokyo 169-8555, Japan

3 Department of Advanced Science Engineering, Waseda University, Japan, 3-4-1 Okubo, Shinjuku-ku, Tokyo 169-8555, Japan

${ }^{4}$ Department of Life Science and Medical Bioscience, Waseda University, 2-2 Wakamatsu-cho, Shinjuku-ku, Tokyo 162-8480, Japan

${ }^{5}$ Institute for Advanced Research of Biosystem Dynamics, Waseda Research Institute for Science and Engineering, Graduate School of Advanced Science and Engineering, Waseda University, 34-1 Okubo, Shinjuku-ku, Tokyo 169-8555, Japan

\begin{abstract}
Mechanism of production of extracellular vesicles (EVs) and their molecular contents are of great interest owing to their diverse roles in biological systems and are far from being completely understood. Even though, cellular cargo release mediated by EVs have been demonstrated in several cases, their role in secondary metabolite production and release remains elusive. In this
\end{abstract}


study we investigate this aspect in detail using Raman micro-spectroscopic imaging. We provide considerable evidence to suggest that the release of antibiotic penicillin by filamentous fungus Penicillium chrysogenum involves EVs. Morphological modifications of the fungal body during biogenesis, changes in cell composition at the locus of biogenesis, and major molecular contents of the released EVs are also revealed in this study.

\section{Importance}

Extracellular vesicles (EVs) play a key role in cellular communications. EVs role in functioning of fungi are relatively less explored. Here we show selective enrichment of chemical contents at certain locations of mycelium of $P$. chrysogenum forming protruding regions. Secondary metabolite penicillin is excessively localized in them. We provide evidence to show that EVs are released from these protrusions. Raman imaging has been applied for molecular profiling of the mycelium and for characterizing chemical contents of the EVs. Our study suggests a possible general role of EVs in the release of antibiotics from the producing organisms.

\section{Keywords}

Raman micro-spectroscopy, extracellular vesicles, fungi, penicillin, secondary metabolite, multivariate curve resolution. 


\section{Introduction}

All cellular systems including microorganisms, multicellular organisms, and even circulating cells in the body fluids of higher organisms, require frequent transmembrane molecular transport to function effectively. The secretion of the molecules in vesicular cargo has received renewed interest originating from their roles in cellular communications (1), viral/bacterial infections $(2$, $3)$, immune system response $(4,5)$, cancer $(6,7)$ etc. Initial studies have attributed the origin of such vesicular cargo units to be mainly endosomal in nature (8). Recently, plasma membrane mediated generation of extracellular vesicles (EVs), with sizes varying from $50 \mathrm{~nm}$ to 1,000 nm, have been observed in several living systems $(2,9)$. EVs' role in pathogenesis is becoming more evident lately, for instance, EVs produced by infectious Staphylococcus aureus delivers the toxin to human host cells (10). Evidence support that bacterial probiotic effects are also mediated through EVs containing effector molecules (e.g. Lactobacillus casei) (11). Inspired by EVs roles in living systems, EVs based therapeutic strategies are also being developed for RNA and protein delivery, and targeted drug delivery (12). Addressing the mechanism of production and the molecular constitution of EVs produced by microorganisms are especially important because of their complex roles in pathogenesis (13) to inventive roles in therapeutics (14).

Mechanism of production of EVs and their functional roles in diverse cellular systems are currently being actively investigated. According to the current understanding, in gram negative bacteria, budding (15) of the outer membrane occurs at the regions having weaker crosslinking with the peptidoglycan (PG) layer (16), and then the EVs detach from the cells without compromising the membrane integrity (17). The degradation of PG layer by a biological agent can also result in explosive cell lysis leading to generation of a large number of EVs (18). In 
bacteria Pseudomonas aeruginosa and Bacillus subtilis the role of endolysins in effecting PG degradation leading to the production of EVs has been demonstrated (18-21). In the case of filamentous fungi, with thicker polysaccharide cell wall and without phospholipid outer membrane, the mechanism of EVs biogenesis remains unclear. Owing to the presence of thick polysaccharide cell wall, with inherent porosity in the range of a few nanometers (19), a larger opening in the cell wall might be necessary for EVs secretion. Cryptococcus neoformans produces virulent polysaccharide glucuronoxylomannan in Golgi secreted vesicles and transport it across cell wall (22). Such fungal EVs secretion has also been demonstrated in Histoplasma capsulatum, Paracoccidioides brasiliensis, and Aspergillus fumigatus (23), however, no cell wall damage/opening has been observed in these cases. Despite diverse mechanisms involved, there is considerable evidence to suggest that EVs generation is a well-regulated biological process (2427) rather than a stochastic event occurring at leaky regions of the membrane.

Since EVs are biologically regulated depending on the functional requirements of the organism, existence of subpopulations of EVs with varying sizes (28), composition (29-31), and contents (32), are possible. However, such properties are rather difficult to explore (24-26). Secondary metabolites produced by microorganisms, including several fungi, are central in human defense against pathogenic infections (33). The production, localization, and the release of these key molecular secretions from the cell body are of considerable interest particularly because of antibiotic resistance prevalent in microbial community $(34,35)$. It is unclear if the release of secondary metabolites involves EVs. In this study, we investigate the possible role of EVs in the release of penicillin produced by $P$. chrysogenum. For a detailed study, we have employed molecular structure specific Raman micro-spectroscopic imaging technique in conjunction with 
multivariate curve resolution (MCR) analysis. Since the antibiotic molecule, penicillin G, is structurally different from common cellular constituents, its localization and a possible release involving EVs can be investigated with this technique. The effectiveness of this methodology has been demonstrated in our recent study by performing molecular profiling of lipid droplets in human liver cells (36). Our modified MCR analysis routine $(36,37)$ quantitatively decomposes highly overlapped composite Raman spectral data into spectra of biomolecules revealing their individual spatial distributions. Owing to the presence of thick polysaccharide layer at the cell boundaries, strong Raman signal specific to polysaccharides can be obtained (38), which will then allow inspecting the integrity of the cell wall in case EVs mediate the release of the antibiotic. The exquisite ability of Raman imaging technique to detect production and localization of penicillin inside mycelia of $P$. chrysogenum has been demonstrated in our recent study (38). Here we investigate the penicillin release by EVs biogenesis from $P$. chrysogenum.

\section{Results}

Penicillin production in the $P$. chrysogenum culture extract was confirmed with antimicrobial activity test (inhibition zone $50 \mathrm{~mm} / 10 \mu \mathrm{l}$ per disk) and LC-MS analysis (Fig. S1). Raman imaging was conducted on the filamentous mycelium and MCR data analysis (see materials and methods) was performed. Results of the MCR analysis are given in Fig. 1. Characteristic spectra corresponding to proteins, lipids, cytochrome c, penicillin, polyphosphate and polysaccharide can be accurately identified (For details of spectral assignments see materials and methods: spectral assignments). Raman images showing spatial distribution of these biomolecules in the mycelium are provided in Fig. 1b. In the Raman images, proteins and lipids can be seen more or 
less continuously distributed throughout the cell. Polyphosphate and cytochrome c are distributed at certain regions of the cell, in agreement with our previous study (38). A protrusion originating from mycelium is indicated with a circle. An overlay of proteins and polysaccharides (Fig. 1c), and a drawing indicating protrusion (Fig. 1d) are also shown. A high concentration of penicillin can be noticed at the protrusion (Fig. 1b).

(Figure 1)

We examined the culture medium to confirm the presence of EVs. Several large $(\sim 1 \mu \mathrm{m})$ EVs were found in the culture medium outside the cell regions (Fig. 2a). It is important to examine the composition of the EVs in order to elucidate the possible role of penicillin in their biogenesis. Raman images of the EVs (Fig. 2a; Fig. S3) and the results of MCR analysis (Fig. 2b) are provided in Fig. 2 a-c. Proteins, penicillin, and carotenoid were detected inside EVs (Fig. 2b), and on an average these EVs contain $\sim 4 \pm 1 \mathrm{mM}$ protein, $\sim 38 \pm 19 \mathrm{mM}$ penicillin and $\sim 5 \pm 5 \mu \mathrm{M}$ carotenoid (Fig. 2c; all concentrations estimated relative to Raman intensities of standard compounds in solution. For details see materials and methods; \pm std. dev.; carotenoid is resonance enhanced). No size dependent variation in penicillin content was observed in the EVs (Fig. 2d). It is important to note that six different molecules were detected in the filamentous cell body, however, only protein, penicillin and carotenoid were detected inside the EVs. Carotenoids were detected in the EVs and not in the main filamentous body of the organism (38). Resonance enhancement makes it possible to detect carotenoid even at very low concentrations $(\mu \mathrm{M})$ using Raman spectroscopy. Based on the observed peak positions, the carotenoid spectral component has been assigned to $\beta$-carotene (39). However, several different carotenoids give Raman spectra 
that are only marginally different from one another and exhibit peak shift associated with mode of binding in biological systems (39). HPLC analysis of the concentrated extract from the $P$. chrysogenum culture (supernatant containing EVs) was performed and the presence of $\beta$ carotene was confirmed (Fig. 2e). Notably, polysaccharide is absent in the EVs (Fig. 1) suggesting these are not broken fragments of the main filamentous body. Owing to the relatively lower concentration of lipids in the EVs membrane, it was not detected in the Raman analysis. In order to verify the presence of lipid membrane surrounding the EVs, we performed staining with a membrane selective dye (FM 4-64; (N-(3-Triethylammoniumpropyl)-4-(6-(4-(Diethylamino) Phenyl) Hexatrienyl) Pyridinium Dibromide)). As expected, the dye has labelled the EVs, which affirms its lipid membrane bound structure (Fig. 2f).

TEM images of the EVs from the supernatant of the culture broth are shown in Fig. 2g. Lighter EVs can be separated from denser cell debris by performing density gradient ultracentrifugation (40). LC-MS analysis was performed to examine the presence of penicillin in different layers of the sample after ultracentrifugation. Significant amount of penicillin was detected in the top two layers (each $2 \mathrm{ml}$ ), while penicillin was not detected in the subsequent layers (Fig. S4). We have performed TEM analysis of these layers and a large number of EVs were detected in the first two layers (Size: $180 \mathrm{~nm}$ to $600 \mathrm{~nm}$ ) while cell debris were seen in the subsequent layers (Fig. S4). This result reaffirms the production of EVs containing penicillin by $P$. chrysogenum.

Our attempts to obtain Raman images of EVs after ultracentrifugation was not successful due to fortuitous ingress of iodixanol (41) into the isolated EVs (Fig. S5). This method must be modified in future work. 
P. chrysogenum is known to produce spores of sizes comparable to that of EVs observed and therefore it is necessary to distinguish the EVs from spores. We have separately cultured spores of $P$. chrysogenum and Raman imaging was conducted. The results of MCR analysis indicated prominent polysaccharide (42) signature (Fig. S6), which was absent in the vesicular structures.

(Figure 2)

$\mathrm{Ca}^{2+}$ is a chemical constituent keeping polysaccharide layer intact in the fungus cell wall. Removal of $\mathrm{Ca}^{2+}$ by adding small amounts of EDTA should weaken the cell wall and thereby it may facilitate the formation of protruding regions (43). We have therefore added EDTA into the culture medium to facilitate the EVs production. As expected, several protrusions were observed in the filamentous body (Fig. S7). The results of the Raman-MCR analysis are given in Fig. 3.

(Figure 3)

Appearance of several protruding regions in the filamentous body suggests that addition of EDTA has facilitated its formation and therefore the biogenesis of the EVs. All these protrusions were rich in penicillin, similar to the protrusions observed in the culture without EDTA. Raman images given in Fig. 3 indicate $\beta$-carotene as one of the constituents of the protrusions (except one). Notably, $\beta$-carotene was not seen as a chemical constituent of the filamentous body. Additionally, $\beta$-carotene was a constituent of all the EVs shown in Fig. 2a. 


\section{Discussion}

The appearance of protrusions in the main filamentous body with characteristically different chemical composition is clearly demonstrated with Raman imaging (Fig. 1b). A comparison of the penicillin and polysaccharide distributions reveals interesting aspects. Penicillin is highly concentrated in the balloon like protrusion (Fig. 1b: penicillin) compared to the main filamentous body (in terms of Raman intensities, $\sim 18 \%$ in the protrusion compared to $\sim 4 \%$ in the mycelium (Fig. 1b)). Interestingly, the protrusion does not have polysaccharide (Fig. 1b: polysaccharide) boundary, and where it originates, the polysaccharide cell wall appears comparatively thin and, apparently, with a small opening (Fig. S7e). Endolysin induced hole formation in the PG layer and consequent EVs release is known to occur (19). However, in the present case no external chemical inducer was employed. If the opening was a physically formed, then there is no reason for penicillin concentration at the protrusion to be considerably different from cytoplasmic composition. A characteristically high concentration of penicillin, we believe, implies a molecular mechanism that involves penicillin itself, and probably an urge to release it. The following scenario is possible. In order to release penicillin, it is first accumulated at specific regions of the filamentous body. A protrusion is then formed through the cell wall, from which, probably, EVs containing penicillin are subsequently released. Supporting this hypothesis, we detected several EVs containing penicillin in the culture supernatant (Fig. 2a and g) with high relative composition of penicillin is observed in the EVs similar to that in the protrusion. Moreover, no polysaccharides were detected in the EVs (hence not spores or fragments of the mycelium) and a lipid selective fluorescent dye has labelled the EVs. Relatively high concentration of penicillin in both the protrusions and the isolated EVs indicates that the production of EVs is linked to the formation of the protrusions. 
EDTA addition has facilitated the formation of protrusions suggesting that a higher porosity (or an opening) in the cell wall is necessary for the biogenesis (EDTA weakens cell wall(43)). Further, $\beta$-carotene is observed in the EVs and in the protrusions but not in the other parts of mycelium (Fig. 3). Therefore, most probably $\beta$-carotene is produced during the biogenesis of the EVs to protect them from possible environmental stress $(44,45)$. Penicillium sp. is known to produce carotenoid under oxidative stress (46). Production of $\beta$-carotene at the protruding regions also supports the fact that the observed event is, most probably, the biogenesis of the EVs. It is also interesting to note that all the protrusions do not have exactly same composition (compare intensities of the circled regions in Fig. 3). In Fig. 3, cytochrome c is seen in two of the protruding regions in spite of their absence in isolated EVs. $\beta$-carotene was also absent in one of the three protrusions (Fig. 3). In the absence of additional evidence, we attribute it to the different stages of biogenesis. Absence of $\beta$-carotene in the protrusion in Fig. 1 could also be reasoned along the similar lines. Further, we find no evidence to suggest that EDTA induces excess $\beta$-carotene production in $P$. chrysogenum.

High penicillin content in the EVs is evident from the Raman images (Fig. 2), which is also supported by LC-MS results (penicillin was detected only in the layers containing EVs; Fig. S6). Taken together, it is clear that the EVs containing relatively larger amount of penicillin are produced by $P$. chrysogenum. During different stages of biogenesis, the protruding regions can have different compositions. The contents of the protrusions are, apparently, progressively regulated, and EVs with similar molecular contents are eventually released by the organism.

Penicillin biosynthesis by $P$. chrysogenum involves multiple enzymatic reactions: a) synthesis of a -(L- $\alpha$-amino adipyl)-L-cysteinyl-D-valine (ACV) tripeptide mediated by ACV synthetase, 
b) $\mathrm{ACV}$ is converted to isopenicillin (IPN) by Isopenicillin N synthase (IPNS) and finally, c) enzymatic conversion of isopenicillin intermediate to penicillin $\mathrm{G}$, either completed in a single enzymatic step involving acyl CoA:IPN acyltransferase, or through a two-step enzymatic reaction involving an intermediate 6-aminopenicillanic acid (6-APA) $(47,48)$. The final step (i.e., step c) of penicillin biosynthesis occurs in specific locations called microbodies (peroxisomes) (48-50), which are of dimensions comparable to the EVs observed in our study (49, 51). Interestingly, increased number of peroxisomes inside filamentous body of $P$. chrysogenum has also been correlated with higher penicillin production $(50,52)$. It is highly probable that the microbodies are one of the major sources of the vesicular (EVs) contents produced by $P$. chrysogenum.

Penicillin release by exocytosis mediated by vacuoles produced by pexophagy of microbodies has been proposed in an earlier study based on electron microscopy images (53). In spite of the difference in the suggested mechanism, our conjecture of relation between contents of microbody and EVs is supported by this study. EVs with heterogeneous molecular contents secreted by fungus Cryptococcus neoformans were earlier observed in the supernatants of the cultures (54). It was hypothesized that these EVs are Golgi synthesized and later brought to near plasma membrane. The mechanism of cell wall passage, however, was not clear. Our results show the generation of EVs though a process involving extrusion of specific cytoplasmic contents though the cell wall, albeit in a different fungus.

Raman spectroscopy, being a molecule specific analytical method, combined with our novel MCR routine $(36,37)$ allowed detection of multiple chemical species inside the filamentous body of $P$. chrysogenum. This exquisite ability has been utilized in the present study to address several important questions concerning the mechanism of biogenesis, and the chemical 
composition of the EVs. Our study provides first consolidated evidence for the release of secondary metabolite - penicillin in the present case - from a fungus mediated by EVs. We show that penicillin is enriched at the regions of the fungal body where the EVs biogenesis occur. Through the polysaccharide cell wall penicillin rich protrusions form, and the contents are then released in the form of EVs. However, it remains unclear from our study whether this is the only mechanism for the release of penicillin. Our findings and suggested mechanism are also supported by the recent study by Toyofuku et al., albeit in Gram-positive bacteria (19). We believe that penicillin accumulation, polysaccharide wall 'opening' and release of EVs are all steps in the major cascade in the biogenesis of the EVs in P. chrysogenum. It is possible that EVs also, in general, mediate the release of secondary metabolites from microorganisms.

\section{Materials and Methods}

\section{Raman Spectroscopy}

Raman microspectroscopic imaging measurements were carried out with a laboratory-built confocal Raman microspectrometer. A $532 \mathrm{~nm}$ output of a Nd:YAG laser (Compass 315M; Coherent Inc., Santa Clara, CA, USA) was used as the laser source. The laser beam was focused with a 100X (1.4 NA) objective lens (Plan Apo VC; Nikon Corporation, Tokyo, Japan) mounted on an inverted microscope (ECLIPSE Ti; Nikon Corporation, Tokyo, Japan). The back-scattered Raman light was measured with a spectrometer (MS3504i, 600 lines/mm; SOL Instruments, Ltd., Minsk, Republic of Belarus) and a CCD detector (Newton DU920-M; Andor Technology Plc., Antrim, UK). The laser power at the sample was $\sim 10 \mathrm{~mW}$. A piezoelectric stage (custom-made; Physik Instrumente GmbH \& Co. KG, Karlsruhe, Germany) was used to carry out Raman imaging $(0.3 \mu \mathrm{m}$ step size) with $1 \mathrm{sec}$ exposure for each Raman spectral acquisition. Spectra 
were calibrated using indene. Raman data analysis and detailed image processing were performed with codes written in Igor Pro. Image J was also used to plot Raman images.

\section{MCR-ALS and SVD}

Multivariate curve resolution (MCR) by alternating least squares (ALS) is performed by solving eqn. 1. Raman data matrix $\left(A_{m, n}\right)$ can be decomposed into spectral components $\left(\mathrm{W}_{\mathrm{m}, \mathrm{k}}\right)$ and their concentration profiles $\left(\mathrm{H}_{\mathrm{k}, \mathrm{n}}\right)(36,37,55-59)$.

$A_{m, n}=W_{m, k} H_{k, n}+E_{m, n}$

$\mathrm{E}$ is the residual. In the present case, $\mathrm{A}_{\mathrm{m}, \mathrm{n}}$ consists of $\mathrm{n}$ spectra from different spatial locations of the mycelium each with $\mathrm{m}$ wavenumber points. MCR-ALS is performed iteratively by minimizing the Frobenius norm $\|\mathrm{A}-\mathrm{WH}\|^{2}$ until negligible residual E results. Non-negativity constraints $\mathrm{W} \geq 0$ and $\mathrm{H} \geq 0$ are applied during the minimization procedure to obtain interpretable solutions because all the Raman spectral intensities and the concentrations are necessarily positive. The value of ' $\mathrm{k}$ ' was guessed at first and later optimized. The number of independent spectral components with dominant singular values in singular value decomposition (SVD) served as an initial guess for ' $k$ '. Sparser MCR solutions were sought, when required, by introducing regularization schemes such as L1 norm (Lasso regression; $\alpha$ ) or L2 norm (Ridge regression; $\beta$ ). L1 norm can be applied to $\mathrm{H}$ matrix or $\mathrm{W}$ matrix depending on situation as given below.

$$
\left(\mathrm{W}^{\mathrm{T}} \mathrm{W}+\alpha^{2} \mathrm{~F}\right) \mathrm{H}=\mathrm{W}^{\mathrm{T}} \mathrm{A}
$$




$$
\left(H H^{\mathrm{T}}+\alpha^{2} \mathrm{~F}\right) \mathrm{W}=\mathrm{HA}^{\mathrm{T}}
$$

Where $\mathrm{F}$ is a $\mathrm{k} \times \mathrm{k}$ matrix in which all its elements are unity.

L2 norm can similarly be applied to $\mathrm{H}$ matrix or $\mathrm{W}$ matrix depending on situation as follows.

$$
\begin{aligned}
& \left(\mathrm{W}^{\mathrm{T}} \mathrm{W}+\beta^{2} \mathrm{I}\right) \mathrm{H}=\mathrm{W}^{\mathrm{T}} \mathrm{A} \\
& \left(\mathrm{HH}^{\mathrm{T}}+\beta^{2} \mathrm{I}\right) \mathrm{W}=\mathrm{HA}^{\mathrm{T}}
\end{aligned}
$$

Where I is an identity matrix.

The optimized H matrix was plotted as a 2D Raman images.

\section{Cell culture}

P. chrysogenum KF-425 was used as a fungus system producing antibiotics. Spores of strain KF425 was inoculated into $10 \mathrm{~mL}$ preculture medium, containing $0.5 \%$ polypeptone, $2 \%$ glucose, $0.2 \%$ yeast extract, $0.1 \% \mathrm{KH}_{2} \mathrm{PO}_{4}$, and $0.05 \% \mathrm{MgSO}_{4} \cdot 7 \mathrm{H}_{2} \mathrm{O}$. It was incubated at $27{ }^{\circ} \mathrm{C}$ with shaking at $200 \mathrm{rpm}$ for 2 days. About $1 \mathrm{ml}$ of this pre-culture was then homogenized and inoculated into $100 \mathrm{~mL}$ main culture (F7; penicillin producing) medium, consisting of $2 \%$ sucrose, $1 \%$ glucose, $3 \%$ corn steep powder, $0.5 \%$ meat extract, $0.05 \% \mathrm{MgSO}_{4} \cdot 7 \mathrm{H}_{2} \mathrm{O}, 0.1 \%$ $\mathrm{KH}_{2} \mathrm{PO}_{4}$, and $0.3 \% \mathrm{CaCO}_{3}$ (adjusted to $\mathrm{pH} \sim 7.3$ before sterilization). The liquid culture was incubated at $27{ }^{\circ} \mathrm{C}$ with shaking at $200 \mathrm{rpm}$ for 5 to 6 days. After this, $1 \mathrm{~mL}$ of the culture broth was taken, $1 \mathrm{ml}$ ethanol was added, and centrifuged at about $10000 \mathrm{rpm}$. HPLC and LC-MS experiments were conducted using the supernatant to confirm penicillin production. An aqueous solution of benzylpenicillin potassium (Penicillin G) (Fujifilm Wako Pure Chemical Co., Tokyo, Japan) was used as the reference compound. An antimicrobial activity test (inhibition zone assay) was also performed, as reported earlier (38), to confirm penicillin production. 
EDTA (Ethylenediaminetetraacetic acid) experiment: On the third day of $P$. chrysogenum culture, $2 \mathrm{ml}$ culture was separated into a new test tube and $100 \mu \mathrm{L}$ of $0.5 \mathrm{M}$ EDTA solution was added, and culturing was continued for another 2 days. Afterwards filamentous $P$. chrysogenum cells were imaged with Raman microspectroscopy

\section{Antibacterial Activity Test}

Antibacterial activity test was conducted using Kocuria rhizophira NBRC 103217. The antimicrobial activity was evaluated by a disk-diffusion agar method using paper disks ( $\varphi 8 \mathrm{~mm})$ soaked with $10 \mu \mathrm{L}$ of $P$. chrysogenum culture extract. The paper disc was placed on an agar plate seeded with $K$. rhizophira. The agar plate was then incubated.

\section{Transmission electron microscopy (TEM)}

TEM images were recorded using JEM-1400 Flash Electron Microscope. For TEM imaging, the P. chrysogenum culture was centrifuged at $3500 \mathrm{rpm}$, filtered through $1 \mu \mathrm{m}$ membrane filter to remove cells and other large debris. The samples were then negatively stained with $1 \%$ uranyl acetate solution and dried on the copper grid. Different layers after ultracentrifugation were also used as samples for detecting EVs.

\section{Gradient ultracentrifugation}

Iodixanol solution (OptiPrep, CosmoBio, Japan; 60\%) was diluted in MQ water to 45\%, 40\%, $35 \%, 30 \%$, and 25\%.(60) These solutions are then layered into a test tube such that the solution with highest density is at the bottom. Layers were frozen after each addition. Sample solution containing EVs was placed at the top of the tube (at RT) and ultracentrifugation (Beckman 
Optima L-90K Ultracentrifuge) was performed at 40,000 RPM for 16 hours at $4^{\circ} \mathrm{C}$. Afterwards, $2 \mathrm{ml}$ layers were separated and analyzed.

\section{Liquid chromatography (LC)-Mass spectroscopy (MS) and HPLC conditions}

Liquid chromatography-high resolution electrospray ionization mass spectrometry (LC/MS) spectra were measured using an AB Sciex TripleTOF 4600 System (AB Sciex, Framingham, MA, USA). Chromatographic method consisted of solvent $\mathrm{A}\left(\mathrm{H}_{2} \mathrm{O}\right.$ with $2 \mathrm{mM}$ ammonium acetate) and B (acetonitrile with $2 \mathrm{mM}$ ammonium acetate), starting at 5\% up to $100 \%$ of B in 10 min followed by a hold of $100 \%$ of B for $5 \mathrm{~min}$, using a CAPCELL CORE C18 $(2.7 \mu \mathrm{m}, 3.0 \times$ $100 \mathrm{~mm}$, Osaka Soda Co. Ltd., Osaka, Japan). The method employed a flow of $0.2 \mathrm{ml} / \mathrm{min}, 2 \mu \mathrm{l}$ injection volume and column temperature of $40^{\circ} \mathrm{C}$. The flow rate was $0.5 \mathrm{ml} / \mathrm{min}$ and the injection volume was $2 \mu \mathrm{l}$. ESI-MS ( $\mathrm{R} \geq 30,000$; tolerance for mass accuracy was about 5 ppm) was recorded for $15 \mathrm{~min}$ in the $\mathrm{m} / \mathrm{z}$ region from 100 to $2000 \mathrm{Da}$.

For identifying the $\beta$-carotene in EVs, $\beta$-carotene extracted with hexane and acetone $(1: 1)$ solvent mixture from culture broth containing EVs of strain KF-425, concentrated the extract. $\beta$ -carotene in the extract was measured using a Nexera Mikros System (Shimadzu Corp., Kyoto, Japan) equipped with SPD-M30A as a photodiode array detector. Chromatographic method consisted of solvent $\mathrm{A}\left(90 \%\right.$ acetonitrile in $\left.\mathrm{H}_{2} \mathrm{O}\right)$ and $\mathrm{B}(100 \%$ ethyl acetate), starting at $0 \%$ up to $50 \%$ of B in 20 min followed by a hold of $100 \%$ of B for 10 min, using a Shin-Pac Velox SPC18 $(2.7 \mu \mathrm{m}, 2.1 \times 150 \mathrm{~mm}$, Shimadzu Corp., Kyoto, Japan). The method employed a flow of $0.2 \mathrm{ml} / \mathrm{min}, 2 \mu \mathrm{l}$ injection volume and column temperature of $40^{\circ} \mathrm{C}$ monitoring at $450 \mathrm{~nm}$.

\section{Spectral assignments}


Polyphosphate: A sharp band at $1160 \mathrm{~cm}^{-1}$ with a broad feature at around $696 \mathrm{~cm}^{-1}$ indicates polyphosphate (61).

Polysaccharides: Two bands in the range 810 to $970 \mathrm{~cm}^{-1}$ are specific to glycosidic linkages in polysaccharides. $\mathrm{C}-\mathrm{H}$ equatorial bending vibrations for $\beta$-type falls in the range $905-885 \mathrm{~cm}^{-1}$ and for $\alpha$ - type between $865-835 \mathrm{~cm}^{-1}(42)$. The broad nature of both the bands indicate complex biochemical nature of the polysaccharide cell wall of the fungus. A band at $1463 \mathrm{~cm}^{-1}$ corresponds to $\mathrm{CH}, \mathrm{CH}_{2}$, and $\mathrm{C}-\mathrm{O}-\mathrm{H}$ deformations of the polysaccharides. Further, broad bands of overlapped peaks centered at around $1360 \mathrm{~cm}^{-1}$ and $1080 \mathrm{~cm}^{-1}$ are characteristic of polysaccharide polymer. These represents $\mathrm{CCH} / \mathrm{COH}$ deformation modes and vibrations of $v(\mathrm{COC})$ glycosidic structures $(62,63)$.

Cytochrome C: A sharp band at $750 \mathrm{~cm}^{-1}$ can be reliably assigned to assigned to pyrrole breathing mode $v_{15}$ in cytochrome c (64). Sharp bands at 1584,1312 and $1129 \mathrm{~cm}^{-1}$ can also be assigned to cytochrome c. The characteristic band at $1312 \mathrm{~cm}^{-1}$ is often regarded as the marker band for cytochrome c (in cytochrome b it appears at $1338 \mathrm{~cm}^{-1}$ ) (65).

Lipids: A band at $1749 \mathrm{~cm}^{-1}$ is due to ester the linkage in lipids. The band at $1657 \mathrm{~cm}^{-1}$ is an indicator of unsaturation in lipids $(\mathrm{C}=\mathrm{C})$. A relatively broad band at $1440 \mathrm{~cm}^{-1}$ is due to- $\mathrm{CH}_{2-}$ scissoring mode (alkyl chain), prominent bands at $1305 \mathrm{~cm}^{-1}(\mathrm{CH}-$ bending) and a broad band $1080 \mathrm{~cm}^{-1}$ corresponding to $\mathrm{C}-\mathrm{C}$ stretching rich in gauche conformation along the carbon chain are all characteristic of lipids $(36,59)$.

Proteins: Prominent protein Raman spectral features include strong amide-I (peptide backbone vibration) at $1665 \mathrm{~cm}^{-1}, \mathrm{C}-\mathrm{H}$ deformation mode at $1450 \mathrm{~cm}^{-1}$, tryptophan $\mathrm{C} \alpha-\mathrm{H}$ deformation at 
$1340 \mathrm{~cm}^{-1}$, phenylalanine at $1003 \mathrm{~cm}^{-1}$. All these prominent features can be seen in the MCR spectral component assigned to proteins $(36,38)$.

Penicillin: Separation of Raman spectrum of penicillin presents a challenge in Raman spectroscopy method because its peaks are highly overlapped with proteins. However, using MCR, owing to its sensitivity to simultaneous variation at multiple wavenumber positions, we could separate proteins from penicillin accurately. Raman spectrum of penicillin has strong vibrational feature at $1005 \mathrm{~cm}^{-1}$ corresponding to benzyl group in penicillin G. A detailed study was performed earlier and thus we confirmed our assignments (38).

$\beta$-carotene: Three strong Raman bands are observed from $\beta$-carotene. A strong band at $1522 \mathrm{~cm}^{-1}$ corresponds to $C=C$ vibrations in a conjugated alkyl chain of $\beta$-carotene. The band at $1154 \mathrm{~cm}^{-1}$ corresponds to $\mathrm{C}-\mathrm{C}$ stretching mode coupled with $\mathrm{C}-\mathrm{H}$ bending and the Raman band corresponding to $\mathrm{C}-\mathrm{CH}_{3}$ rocking mode appears at $1001 \mathrm{~cm}^{-1}(39)$.

\section{Estimating composition of EVs}

In order to estimate the composition of EVs, standard curves (Raman intensity vs. concentration) for benzylpenicillin potassium (in water), B-carotene (in ethanol), and BSA (30\% w/v; for proteins in EVs) were made following the standard procedures. Standard solutions of five different concentrations were prepared in each case: from $118.7 \mathrm{mM}$ to $47.5 \mathrm{mM}$ for benzylpenicillin potassium, 4.5 to $1.5 \mathrm{mM}$ for $\mathrm{BSA}$, and $24.2 \mu \mathrm{M}$ to $8.1 \mu \mathrm{M}$ for $\beta$-carotene. Experimental conditions for the Raman measurements were similar to that used for Raman imaging of EVs. 


\section{Acknowledgements}

Authors thank Dr. Hiroshi Sagara and Dr. Yuji Watanabe, Institute of medical science, University of Tokyo for their help in recording TEM images. This work was supported by JSPS KAKENHI grant number JP17H06158.

\section{Author Contributions}

A.Z.S. and H.T. conceived the idea and designed the study. A.Z.S. conducted Raman measurements, MCR-ALS data analysis, interpretation, image analysis, manuscript writing. A.Z.S, S.H. and T. N. performed penicillium culture, HPLC analysis, LC-MS analysis. A.Z.S. and N.S. performed ultracentrifugation. MCR-ALS codes written by M.A. was used in the study. A.Z.S. and H.T. has contributed to manuscript writing and discussions.

\section{Corresponding author}

Correspondence to Haruko Takeyama.

\section{References}

1. Maacha S, Bhat AA, Jimenez L, Raza A, Haris M, Uddin S, Grivel JC. 2019. Extracellular vesicles-mediated intercellular communication: Roles in the tumor microenvironment and anti-cancer drug resistance. Mol Cancer 18:1-16.

2. Liu Y, Defourny KAY, Smid EJ, Abee T. 2018. Gram-positive bacterial extracellular vesicles and their impact on health and disease. Front Microbiol https://doi.org/10.3389/fmicb.2018.01502.

3. Rodrigues M, Fan J, Lyon C, Wan M, Hu Y. 2018. Role of extracellular vesicles in viral and bacterial infections: Pathogenesis, diagnostics, and therapeutics. Theranostics. 
4. Colombo M, Raposo G, Théry C. 2014. Biogenesis, secretion, and intercellular interactions of exosomes and other extracellular vesicles. Annu Rev Cell Dev Biol 30:255289.

5. Raposo G, Nijman HW, Stoorvogel W, Leijendekker R, Harding C V., Melief CJM, Geuze HJ. 1996. B lymphocytes secrete antigen-presenting vesicles. J Exp Med https://doi.org/10.1084/jem.183.3.1161.

6. Wolfers J, Lozier A, Raposo G, Regnault A, Théry C, Masurier C, Flament C, Pouzieux S, Faure F, Tursz T, Angevin E, Amigorena S, Zitvogel L. 2001. Tumor-derived exosomes are a source of shared tumor rejection antigens for CTL cross-priming. Nat Med 7:297-303.

7. Zhang L, Zhang S, Yao J, Lowery FJ, Zhang Q, Huang WC, Li P, Li M, Wang X, Zhang C, Wang H, Ellis K, Cheerathodi M, McCarty JH, Palmieri D, Saunus J, Lakhani S, Huang S, Sahin AA, Aldape KD, Steeg PS, Yu D. 2015. Microenvironment-induced PTEN loss by exosomal microRNA primes brain metastasis outgrowth. Nature https://doi.org/10.1038/nature15376.

8. Johnstone RM, Adam M, Hammond JR, Orr L, Turbide C. 1987. Vesicle formation during reticulocyte maturation. Association of plasma membrane activities with released vesicles (exosomes). J Biol Chem.

9. Brown L, Wolf JM, Prados-Rosales R, Casadevall A. 2015. Through the wall: Extracellular vesicles in Gram-positive bacteria, mycobacteria and fungi. Nat Rev Microbiol.

10. Thay B, Wai SN, Oscarsson J. 2013. Staphylococcus aureus $\alpha$-Toxin-Dependent Induction of Host Cell Death by Membrane-Derived Vesicles. PLoS One https://doi.org/10.1371/journal.pone.0054661. 
11. Rubio APD, Martínez JH, Casillas DCM, Leskow FC, Piuri M, Pérez OE. 2017. Lactobacillus casei BL23 produces microvesicles carrying proteins that have been associated with its probiotic effect. Front Microbiol https://doi.org/10.3389/fmicb.2017.01783.

12. Murphy DE, de Jong OG, Brouwer M, Wood MJ, Lavieu G, Schiffelers RM, Vader P. 2019. Extracellular vesicle-based therapeutics: natural versus engineered targeting and trafficking. Exp Mol Med.

13. Rivera J, Cordero RJB, Nakouzi AS, Frases S, Nicola A, Casadevall A. 2010. Bacillus anthracis produces membrane-derived vesicles containing biologically active toxins. Proc Natl Acad Sci U S A https://doi.org/10.1073/pnas.1008843107.

14. György B, Hung ME, Breakefield XO, Leonard JN. 2015. Therapeutic applications of extracellular vesicles: Clinical promise and open questions. Annu Rev Pharmacol Toxicol $55: 439-464$.

15. Work E, Knox KW, Vesk M. 1966. THE CHEMISTRY AND ELECTRON MICROSCOPY OF AN EXTRACELLULAR LIPOPOLYSACCHARIDE FROM ESCHERICHIA COLI. Ann N Y Acad Sci https://doi.org/10.1111/j.1749-6632.1966.tb52382.x.

16. Moon DC, Choi CH, Lee JH, Choi CW, Kim HY, Park JS, Kim S II, Lee JC. 2012. Acinetobacter baumannii outer membrane protein a modulates the biogenesis of outer membrane vesicles. J Microbiol 50:155-160.

17. Schwechheimer C, Kuehn MJ. 2015. Outer-membrane vesicles from Gram-negative bacteria: Biogenesis and functions. Nat Rev Microbiol 13:605-619.

18. Turnbull L, Toyofuku M, Hynen AL, Kurosawa M, Pessi G, Petty NK, Osvath SR, CárcamoOyarce G, Gloag ES, Shimoni R, Omasits U, Ito S, Yap X, Monahan LG, Cavaliere R, Ahrens 
CH, Charles IG, Nomura N, Eberl L, Whitchurch CB. 2016. Explosive cell lysis as a

mechanism for the biogenesis of bacterial membrane vesicles and biofilms. Nat Commun https://doi.org/10.1038/ncomms11220.

19. Toyofuku M, Cárcamo-Oyarce G, Yamamoto T, Eisenstein F, Hsiao CC, Kurosawa M, Gademann K, Pilhofer M, Nomura N, Eberl L. 2017. Prophage-triggered membrane vesicle formation through peptidoglycan damage in Bacillus subtilis. Nat Commun https://doi.org/10.1038/s41467-017-00492-w.

20. Toyofuku M, Nomura N, Eberl L. 2019. Types and origins of bacterial membrane vesicles. Nat Rev Microbiol 17:13-24.

21. Resch U, Tsatsaronis JA, Le Rhun A, Stübiger G, Rohde M, Kasvandik S, Holzmeister S, Tinnefeld P, Nyunt Wai S, Charpentier E. 2016. A two-component regulatory system impacts extracellular membrane-derived vesicle production in group a streptococcus. MBio https://doi.org/10.1128/mBio.00207-16.

22. Rodrigues ML, Nimrichter L, Oliveira DL, Frases S, Miranda K, Zaragoza O, Alvarez M, Nakouzi A, Feldmesser M, Casadevall A. 2007. Vesicular polysaccharide export in Cryptococcus neoformans is a eukaryotic solution to the problem of fungal trans-cell wall transport. Eukaryot Cell 6:48-59.

23. Rodrigues ML, Franzen AJ, Nimrichter L, Miranda K. 2013. Vesicular mechanisms of traffic of fungal molecules to the extracellular space. Curr Opin Microbiol 16:414-420.

24. Schwechheimer C, Kulp A, Kuehn MJ. 2014. Modulation of bacterial outer membrane vesicle production by envelope structure and content. BMC Microbiol 14.

25. Roden JA, Wells DH, Chomel BB, Kasten RW, Koehler JE. 2012. Hemin binding protein C is 
found in outer membrane vesicles and protects bartonella henselae against toxic concentrations of hemin. Infect Immun 80:929-942.

26. Tashiro Y, Inagaki A, Shimizu M, Ichikawa S, Takaya N, Nakajima-Kambe T, Uchiyama H, Nomura N. 2011. Characterization of phospholipids in membrane vesicles derived from Pseudomonas aeruginosa. Biosci Biotechnol Biochem 75:605-607.

27. Elhenawy W, Bording-Jorgensen M, Valguarnera E, Haurat MF, Wine E, Feldman MF. 2016. LPS remodeling triggers formation of outer membrane vesicles in salmonella. MBio https://doi.org/10.1128/mBio.00940-16.

28. Murphy K, Park AJ, Hao Y, Brewer D, Lam JS, Khursigaraa CM. 2014. Influence of O polysaccharides on biofilm development and outer membrane vesicle biogenesis in Pseudomonas aeruginosa PAO1. J Bacteriol https://doi.org/10.1128/JB.01463-13.

29. Haraszti RA, Didiot MC, Sapp E, Leszyk J, Shaffer SA, Rockwell HE, Gao F, Narain NR, DiFiglia M, Kiebish MA, Aronin N, Khvorova A. 2016. High-resolution proteomic and lipidomic analysis of exosomes and microvesicles from different cell sources. J Extracell Vesicles https://doi.org/10.3402/jev.v5.32570.

30. Boilard E. 2018. Extracellular vesicles and their content in bioactive lipid mediators: More than a sack of microRNA. J Lipid Res.

31. Llorente A, Skotland T, Sylvänne T, Kauhanen D, Róg T, Orłowski A, Vattulainen I, Ekroos K, Sandvig K. 2013. Molecular lipidomics of exosomes released by PC-3 prostate cancer cells. Biochim Biophys Acta - Mol Cell Biol Lipids https://doi.org/10.1016/j.bbalip.2013.04.011.

32. Zhang H, Freitas D, Kim HS, Fabijanic K, Li Z, Chen H, Mark MT, Molina H, Martin AB, 
Bojmar L, Fang J, Rampersaud S, Hoshino A, Matei I, Kenific CM, Nakajima M, Mutvei AP,

Sansone P, Buehring W, Wang H, Jimenez JP, Cohen-Gould L, Paknejad N, Brendel M,

Manova-Todorova K, Magalhães A, Ferreira JA, Osório H, Silva AM, Massey A, Cubillos-

Ruiz JR, Galletti G, Giannakakou P, Cuervo AM, Blenis J, Schwartz R, Brady MS, Peinado H,

Bromberg J, Matsui H, Reis CA, Lyden D. 2018. Identification of distinct nanoparticles and subsets of extracellular vesicles by asymmetric flow field-flow fractionation. Nat Cell Biol https://doi.org/10.1038/s41556-018-0040-4.

33. O’Brien J, Wright GD. 2011. An ecological perspective of microbial secondary metabolism. Curr Opin Biotechnol.

34. Wilson MC, Mori T, Rückert C, Uria AR, Helf MJ, Takada K, Gernert C, Steffens UAE, Heycke N, Schmitt S, Rinke C, Helfrich EJN, Brachmann AO, Gurgui C, Wakimoto T, Kracht M, Crüsemann M, Hentschel U, Abe I, Matsunaga S, Kalinowski J, Takeyama H, Piel J. 2014. An environmental bacterial taxon with a large and distinct metabolic repertoire. Nature https://doi.org/10.1038/nature12959.

35. Zarins-Tutt JS, Barberi TT, Gao H, Mearns-Spragg A, Zhang L, Newman DJ, Goss RJM. 2016. Prospecting for new bacterial metabolites: A glossary of approaches for inducing, activating and upregulating the biosynthesis of bacterial cryptic or silent natural products. Nat Prod Rep.

36. Samuel AZ, Miyaoka R, Ando M, Gaebler A, Thiele C, Takeyama H. 2020. Molecular profiling of lipid droplets inside HuH7 cells with Raman micro-spectroscopy. Commun Biol 3.

37. Samuel AZ, Horii S, Ando M, Takeyama H. 2021. Deconstruction of Obscure Features in 
SVD-Decomposed Raman Images from P. chrysogenum Reveals Complex Mixing of Spectra from Five Cellular Constituents. Anal Chem 93:12139-12146.

38. Horii S, Ando M, Samuel AZ, Take A, Nakashima T, Matsumoto A, Takahashi Y, Takeyama H. 2020. Detection of Penicillin G Produced by Penicillium chrysogenum with Raman Microspectroscopy and Multivariate Curve Resolution-Alternating Least-Squares Methods . J Nat Prod https://doi.org/10.1021/acs.jnatprod.0c00214.

39. de Oliveira VE, Castro H V., Edwards HGM, de Oliveiraa LFC. 2010. Carotenes and carotenoids in natural biological samples: A Raman spectroscopic analysis. J Raman Spectrosc 41:642-650.

40. Graham J, Ford T, Rickwood D. 1994. The Preparation of Subcellular Organelles from Mouse Liver in Self-Generated Gradients of lodixanol. Anal Biochem 220:367-373.

41. Priebe H, Dugstad H, Gacek M, Hagen E, Homestad OM, Larsen A, Sjogren CE, Thomassen T. 1995. Synthesis and characterization of iodixanol. Acta Radiol Suppl 399:21-31.

42. Noothalapati H, Sasaki T, Kaino T, Kawamukai M, Ando M, Hamaguchi HO, Yamamoto T. 2016. Label-free Chemical Imaging of Fungal Spore Walls by Raman Microscopy and Multivariate Curve Resolution Analysis. Sci Rep https://doi.org/10.1038/srep27789.

43. Baumgarten $T$, Sperling S, Seifert J, von Bergen M, Steiniger F, Wick LY, Heipieper HJ. 2012. Membrane vesicle formation as a multiple-stress response mechanism enhances pseudomonas putida DOT-T1E cell surface hydrophobicity and biofilm formation. Appl Environ Microbiol 78:6217-6224.

44. Venil CK, Velmurugan P, Dufossé L, Devi PR, Ravi AV. 2020. Fungal pigments: Potential coloring compounds for wide ranging applications in textile dyeing. J Fungi 6. 
45. Avalos J, Carmen Limón M. 2015. Biological roles of fungal carotenoids. Curr Genet https://doi.org/10.1007/s00294-014-0454-x.

46. Li XL, Cui XH, Han JR. 2006. Sclerotial biomass and carotenoid yield of Penicillium sp. PT95 under oxidative growth conditions and in the presence of antioxidant ascorbic acid. J Appl Microbiol 101:725-731.

47. Kurzatkowski W, Staniszewska M, Bondaryk M, Gebska-Kuczerowska A. 2014.

Compartmentalization in Penicillin G biosynthesis by Penicillium chrysogenum PQ-96.

Polish J Microbiol 63:399-408.

48. Alvarez E, Cantoral JM, Barredo JL, Diez B, Martin JF. 1987. Purification to homogeneity and characterization of acyl coenzyme A:6-aminopenicillanic acid acyltransferase of Penicillium chrysogenum. Antimicrob Agents Chemother 31:1675-1682.

49. Van Den Berg MA, Albang R, Albermann K, Badger JH, Daran JM, M Driessen AJ, GarciaEstrada C, Fedorova ND, Harris DM, Heijne WHM, Joardar V, W Kiel JAK, Kovalchuk A, Martín JF, Nierman WC, Nijland JG, Pronk JT, Roubos JA, Van Der Klei IJ, Van Peij NNME, Veenhuis M, Von Döhren H, Wagner C, Wortman J, Bovenberg RAL. 2008. Genome sequencing and analysis of the filamentous fungus Penicillium chrysogenum. Nat Biotechnol 26:1161-1168.

50. Muller WH, Van Der Krift TP, Krouwer AJJ, Wosten HAB, Van Der Voort LHM, Smaal EB, Verkleij AJ. 1991. Localization of the pathway of the penicillin biosynthesis in Penicillium chrysogenum. EMBO J 10:489-495.

51. Opaliński L, Bartoszewska M, Fekken S, Liu H, de Boer R, van der Klei I, Veenhuis M, Kiel JAKW. 2012. De novo peroxisome biogenesis in penicillium chrysogenum is not 
dependent on the pex11 family members or pex16. PLoS One 7.

52. Kiel JAKW, Van Der Klei IJ, Van Den Berg MA, Bovenberg RAL, Veenhuis M. 2005.

Overproduction of a single protein, Pc-Pex11p, results in 2-fold enhanced penicillin production by Penicillium chrysogenum. Fungal Genet Biol

https://doi.org/10.1016/j.fgb.2004.10.010.

53. Kurzatkowski W, Gębska-Kuczerowska A. 2016. Pexophagy in penicillin G secretion by Penicillium chrysogenum PQ-96. Polish J Microbiol 65:365-368.

54. Rodrigues ML, Nimrichter L, Oliveira DL, Frases S, Miranda K, Zaragoza O, Alvarez M, Nakouzi A, Feldmesser M, Casadevall A. 2007. Vesicular polysaccharide export in Cryptococcus neoformans is a eukaryotic solution to the problem of fungal trans-cell wall transport. Eukaryot Cell 6:48-59.

55. Ando M, Hamaguchi H. 2013. Molecular component distribution imaging of living cells by multivariate curve resolution analysis of space-resolved Raman spectra. J Biomed Opt https://doi.org/10.1117/1.jbo.19.1.011016.

56. Lee DD, Seung HS. 1999. Learning the parts of objects by non-negative matrix factorization. Nature https://doi.org/10.1038/44565.

57. Hamilton JC, Gemperline PJ. 1990. Mixture analysis using factor analysis. II: Self-modeling curve resolution. J Chemom https://doi.org/10.1002/cem.1180040103.

58. Felten J, Hall H, Jaumot J, Tauler R, De Juan A, Gorzsás A. 2015. Vibrational spectroscopic image analysis of biological material using multivariate curve resolution-alternating least squares (MCR-ALS). Nat Protoc https://doi.org/10.1038/nprot.2015.008.

59. Samuel AZ, Zhou M, Ando M, Mueller R, Liebert T, Heinze T, Hamaguchi H-O. 2016. 
Determination of Percent Crystallinity of Side-Chain Crystallized Alkylated-Dextran

Derivatives with Raman Spectroscopy and Multivariate Curve Resolution. Anal Chem 88.

60. Chutkan H, MacDonald I, Manning A, Kuehn MJ. 2013. Quantitative and qualitative preparations of bacterial outer membrane vesicles. Methods Mol Biol https://doi.org/10.1007/978-1-62703-245-2_16.

61. Naito Y, Toh-e A, Hamaguchi HO. 2005. In vivo time-resolved Raman imaging of a spontaneous death process of a single budding yeast cell. J Raman Spectrosc 36:837-839.

62. Almeida MR, Alves RS, Nascimbem LBLR, Stephani R, Poppi RJ, De Oliveira LFC. 2010.

Determination of amylose content in starch using Raman spectroscopy and multivariate calibration analysisAnalytical and Bioanalytical Chemistry.

63. Chylińska M, Szymańska-Chargot M, Zdunek A. 2014. Imaging of polysaccharides in the tomato cell wall with Raman microspectroscopy. Plant Methods https://doi.org/10.1186/1746-4811-10-14.

64. Okada M, Smith NI, Palonpon AF, Endo H, Kawata S, Sodeoka M, Fujita K. 2012. Labelfree Raman observation of cytochrome c dynamics during apoptosis. Proc Natl Acad Sci U S A 109:28-32.

65. Kakita M, Okuno M, Hamaguchi HO. 2013. Quantitative analysis of the redox states of cytochromes in a living L929 (NCTC) cell by resonance Raman microspectroscopy. J Biophotonics 6:256-259. 
FIG. 1. EVs budding. a) MCR separated Raman spectral components. A full list is provided in the Fig. S2. b) Raman images of chemical constituents of P. chrysogenum filamentous body. The protrusion from the cell body is circled. An apparent location of a small opening in the cell wall is indicated with a white arrow. Size of the small opening cannot be accurately estimated (apparently $<<1 \mu \mathrm{m}$ ) from the image due to the limited spatial resolution of the technique. Scale bar $5 \mu \mathrm{m}$. c) Overlay of polysaccharide and proteins images: red color - polysaccharide and green color - proteins. d) A drawing explaining the observations. The black line indicates polysaccharide cell wall, and the protrusion is shown in green color.

FIG. 2. EVs observation and their characterization. a) white light and Raman images (overlay of $\beta$-carotene, proteins, and penicillin Raman images; for individual images see Fig. S3) of EVs from $P$. chrysogenum observed in the culture. Scale bar $1 \mu \mathrm{m}$. b) molecular constituents of the EVs imaged with Raman microspectroscopy. Molecular compositions of the EVs are characteristically different from that of filamentous body of the organism. c) estimated concentrations of molecular contents of the EVs. d) TEM images of the EVs. Scale bar $200 \mathrm{~nm}$. e) Variation of penicillin content depending on the size of EVs. f) Results of HPLC analysis of concentrated supernatant containing EVs separated from 10 duplicate $P$. chrysogenum cultures. Elution profiles obtained by monitoring at $450 \mathrm{~nm}$ wavelength. UV absorption spectra of standard $\beta$-carotene solution, and $\beta$-carotene in supernatant extract (inset). h) Fluorescence image of an EVs stained with FM 4-64 membrane selective dye. Intensity profile along the line indicated in the fluorescence image is also shown. Fluorescence imaging was performed with the same Raman microscope used in the study.

FIG. 3. Biogenesis of EVs. a) Raman images of EDTA treated P. chrysogenum. Protrusions from the cell wall can be seen at several regions, and all of them have high concentration of penicillin. b) An overlay of polysaccharides and penicillin; it indicates the absence of 
polysaccharides in the protruding regions. c) An overlay of polysaccharide, $\beta$-carotene and penicillin: Apparently, $\beta$-carotene is present exclusively in the protrusions. Scale bar $5 \mu \mathrm{m}$. For the corresponding spectral components, overlay with white light image, Raman image of lipids etc. see fig. S7. 


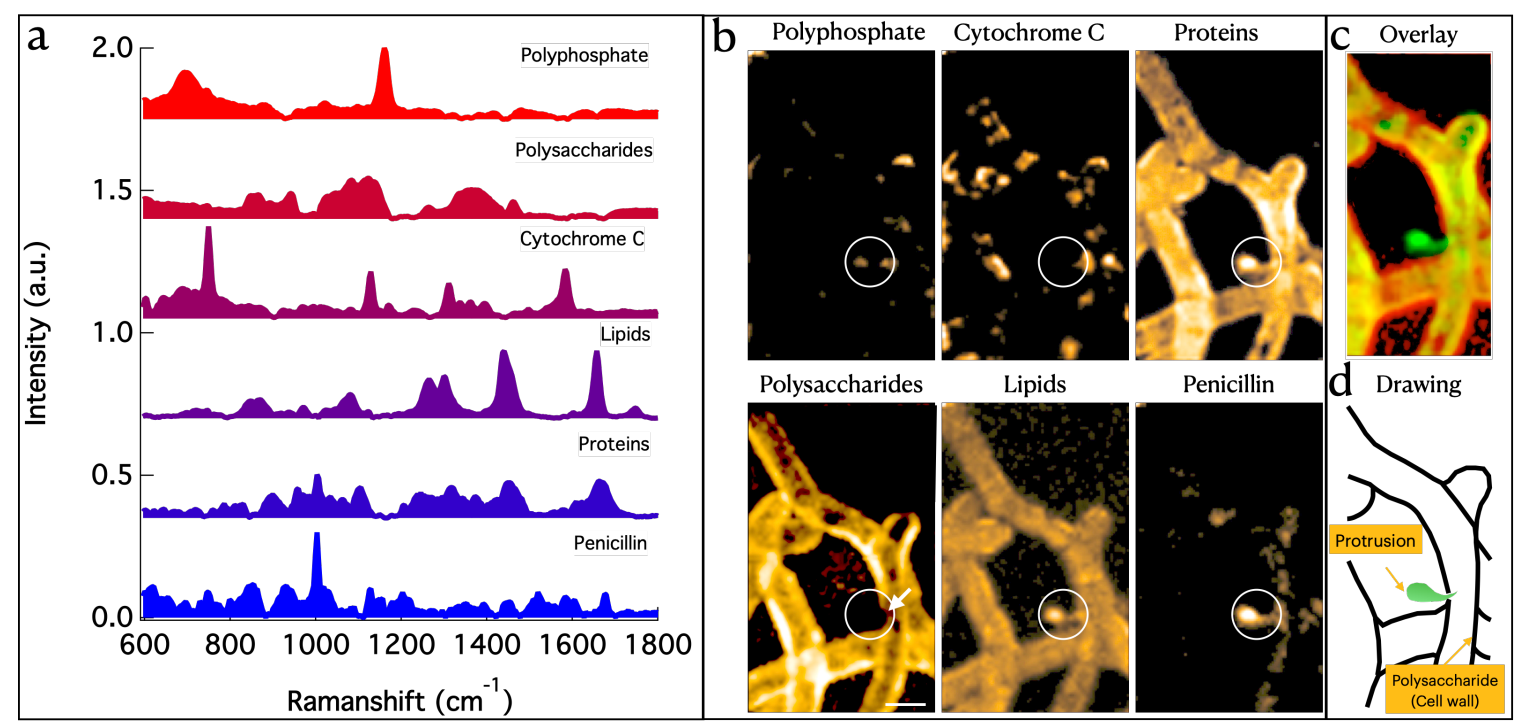

FIG. 1. EVs budding. a) MCR separated Raman spectral components. A full list is provided in the Fig. S2. b) Raman images of chemical constituents of P. chrysogenum filamentous body. The protrusion from the cell body is circled. An apparent location of a small opening in the cell wall is indicated with a white arrow. Size of the small opening cannot be accurately estimated (apparently $<<1 \mu \mathrm{m}$ ) from the image due to the limited spatial resolution of the technique. Scale bar $5 \mu \mathrm{m} . \mathrm{c}$ ) Overlay of polysaccharide and proteins images: red color - polysaccharide and green color proteins. d) A drawing explaining the observations. The black line indicates polysaccharide cell wall, and the protrusion is shown in green color. 


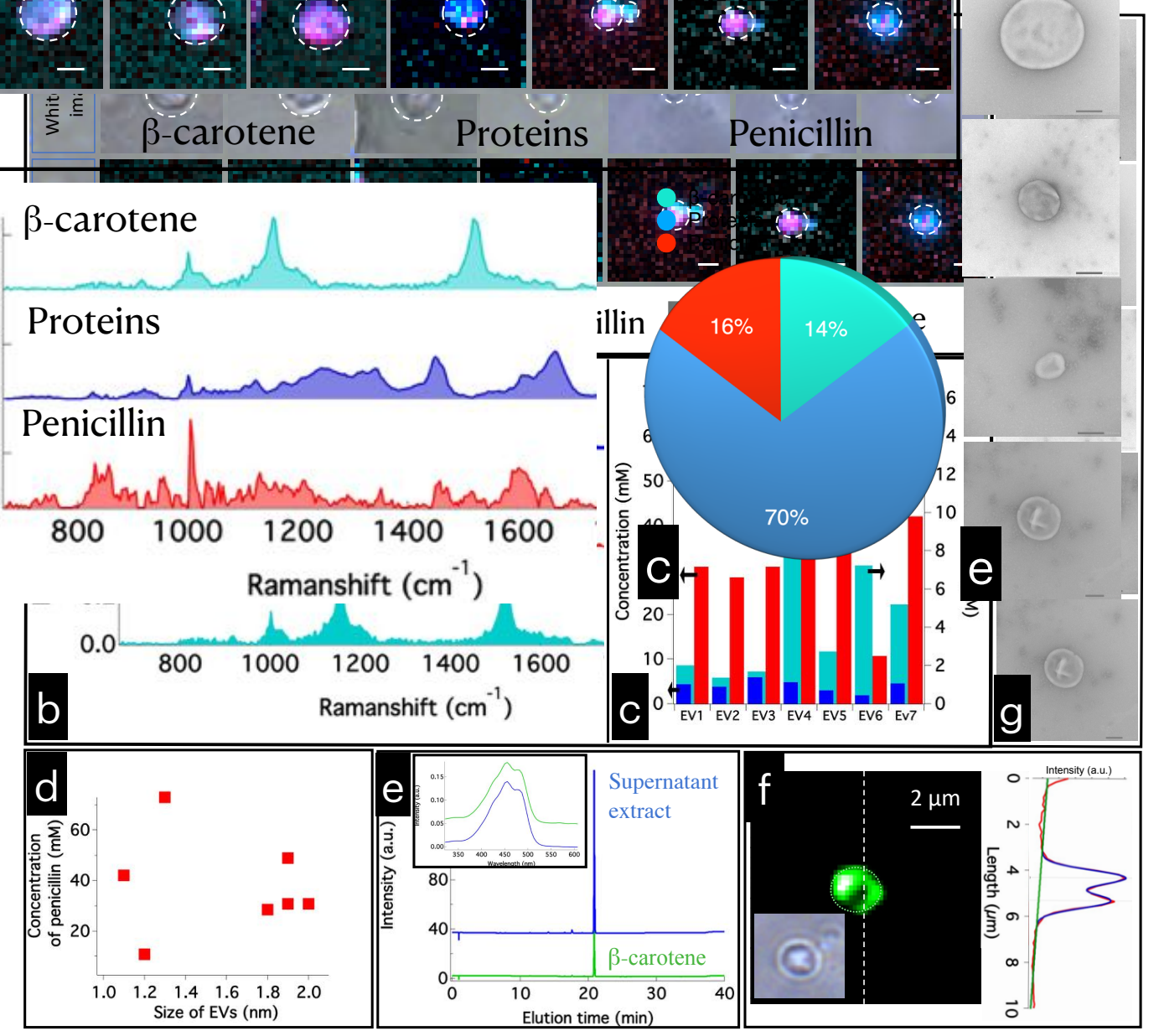

FIG. 2. EVs observation and their characterization. a) white light and Raman images (overlay of $\beta$-carotene, proteins, and penicillin Raman images; for individual images see Fig. S3) of EVs from $P$. chrysogenum observed in the culture. Scale bar $1 \mu \mathrm{m}$. b) molecular constituents of the EVs imaged with Raman microspectroscopy. Molecular compositions of the EVs are characteristically different from that of filamentous body of the organism. c) estimated concentrations of molecular contents of the EVs. d) Variation of penicillin content depending on the size of EVs. e) Results of HPLC analysis of concentrated supernatant containing EVs separated from 10 duplicate $P$. chrysogenum cultures. Elution profiles obtained by monitoring at $450 \mathrm{~nm}$ wavelength. UV absorption spectra of standard $\beta$-carotene solution, and $\beta$-carotene in supernatant extract (inset). f) Fluorescence image of an EVs stained with FM 4-64 membrane selective dye. Intensity profile along the line indicated in the fluorescence image is also shown. Fluorescence imaging was performed with the same Raman microscope used in the study. g) TEM images of the EVs. Scale bar $200 \mathrm{~nm}$. 


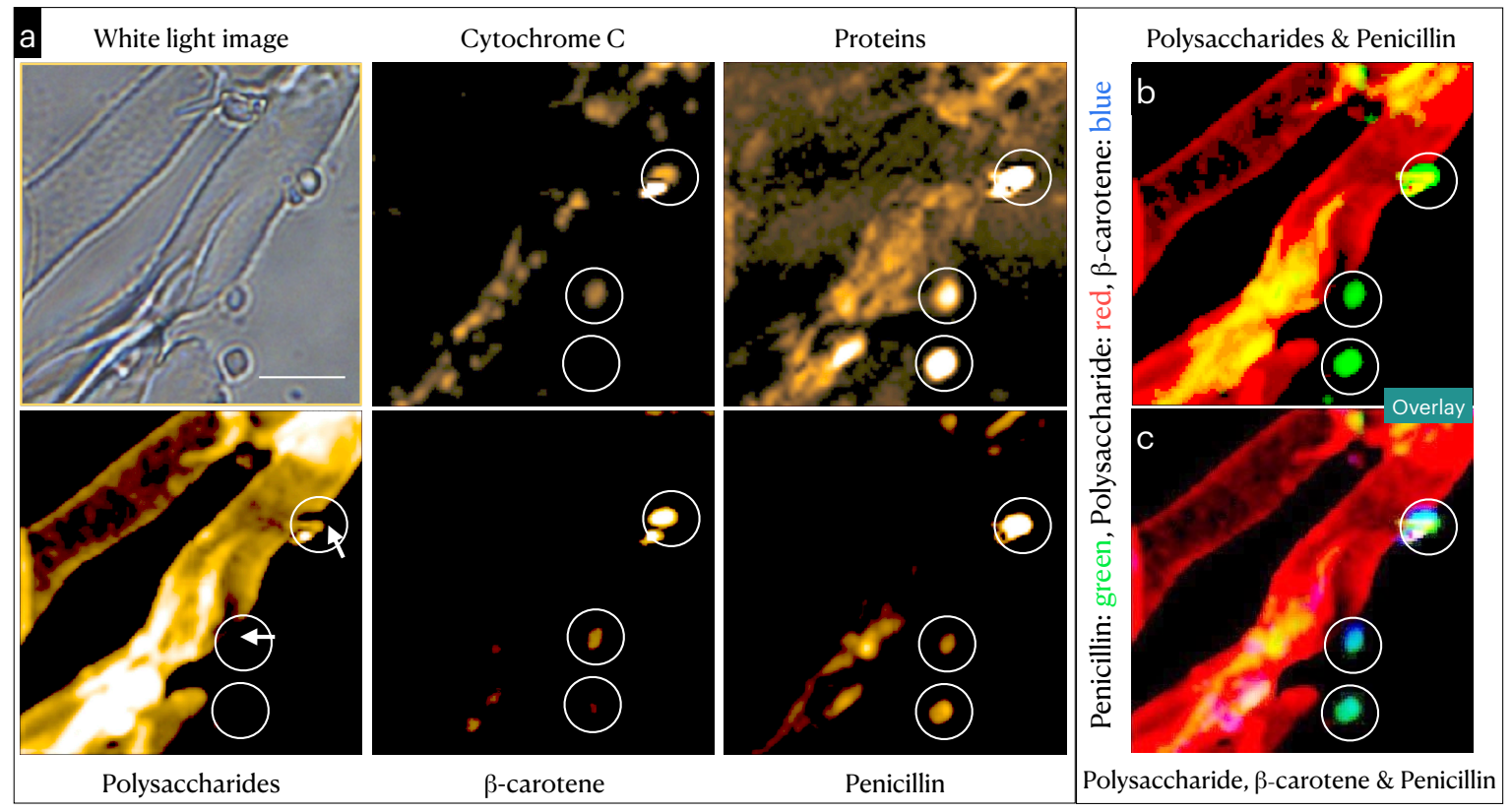

FIG. 3. Biogenesis of EVs. a) Raman images of EDTA treated $P$. chrysogenum. Protrusions from the cell wall can be seen at several regions, and all of them have high concentration of penicillin. b) An overlay of polysaccharides and penicillin; it indicates the absence of polysaccharides in the protruding regions. c) An overlay of polysaccharide, $\beta$-carotene and penicillin: Apparently, $\beta$ carotene is present exclusively in the protrusions. Scale bar $5 \mu \mathrm{m}$. For the corresponding spectral components, overlay with white light image, Raman image of lipids etc. see fig. S7. 